\title{
"Ahí voy a estar hasta que Dios me preste vida". Mujeres en grupos de Alcohólicos Anónimos durante la emergencia sanitaria por el COVID- I9
}

\author{
Nancy Araceli Méndez Romero', Martha Patricia Romero Mendozaㅁ2, Addis Abeba Salinas Urbina(D)3 \\ I Universidad Autónoma Metropolitana Xochimilco, Ciudad de México \\ 2 Instituto Nacional de Psiquiatría Ramón de la Fuente Muñiz, Ciudad de México \\ 3 Departamento de Atención a la Salud. Universidad Autónoma Metropolitana Xochimilco, Ciudad de México
}

\section{RESUMEN}

Introducción: las mujeres han incrementado el consumo de alcohol, lo cual repercute en su bienestar; a ello se agrega la situación de rezago que enfrentan para recibir tratamiento especializado y que las orilla a atender sus padecimientos en grupos de ayuda mutua, escenario que incide en desigualdades sociales y de género. La pandemia de COVID-19 exacerba estas condiciones, afectando situaciones concretas de su vida y su salud. Objetivo: comprender la experiencia de las mujeres que reciben apoyo para su recuperación debido a problemas por el consumo de alcohol en agrupaciones de Alcohólicos Anónimos en el contexto del COVID-19. Método: estudio cualitativo. El muestreo por oportunidad definió la participación de cuatro mujeres de "AA". Durante mayo de 2020, se realizaron entrevistas telefónicas bajo consentimiento informado; se elaboró un análisis temático y se utilizó Atlas.ti. La perspectiva en salud colectiva y de género guiaron el análisis. Resultados: al analizar la información se identificaron tres categorías, la primera contextualiza las experiencias de recuperación de las mujeres; las siguientes categorías develan las situaciones vividas ante la pandemia y el distanciamiento físico. Los hallazgos fueron: los roles y estereotipos de género se reafirman expresando el hostigamiento y descrédito; se incrementan cargas familiares y preocupaciones; la participación en su recuperación disminuyó. Discusión y conclusiones: en el contexto del COVID-19 bajó la participación de las mujeres de "AA" en su recuperación, lo cual pone en riesgo su bienestar, a pesar de que organismos internacionales recomendaron continuar la atención de personas con problemas de salud mental e incorporar acciones comunitarias.

Palabras clave: COVID-19, mujeres, alcohol, género.

\begin{abstract}
Introduction: women have increased the consumption of alcohol, affecting their well-being, to this is added the situation of lag they face in receiving specialized treatment, treating their ailments in mutual-help groups, this scenario affects social and gender inequalities. The COVID-19 pandemic exacerbates these conditions, affecting specific life situations of women and their health. Objective: to understand the experience of women who receive support for their recovery due to problems due to alcohol consumption in groups of Alcoholics Anonymous in the context of COVID-19. Method: qualitative study. Sampling by opportunity defined the participation of four women from "AA". During May 2020, telephone interviews were conducted under informed consent. A thematic analysis was developed and Atlas.ti was used. The perspective on collective health and gender guided the analysis. Results: when analyzing the information, three categories were identified. The first contextualizes the recovery experiences of women. The following categories reveal the situations experienced in the face of the COVID-19 pandemic and physical distancing. The findings were: gender roles and stereotypes are reaffirmed by expressing harassment and discrediting; family burdens and worries increase; participation in its recovery decreased. Discussion and conclusions: in the context of COVID-19, the participation of AA women in their recovery decreased, putting their well-being at risk, despite the fact that international organizations recommended continuing the care of people with mental health problems and incorporating community actions.
\end{abstract}

Keywords: COVID-19, women, alcohol, gender.

\footnotetext{
Autor de correspondencia:

Nancy Araceli Méndez Romero. Celular: 5516569298

Correo electrónico: nmr2500@yahoo.com.mx

Recibido: 22 de septiembre de 2020

Aceptado: 22 de octubre de 2020

doi: 10.28931/riiad.2020.2.05
} 


\section{INTRODUCCIÓN}

La pandemia por el virus SARS-CoV-2, causante de la enfermedad del COVID-19, ha trastocado la vida de las personas y sus colectivos. En México, las desigualdades e inequidades sociales preexistentes se han ido profundizado dada la emergencia sanitaria (Boichat, 2020). Las desigualdades son producto de condiciones históricas, económicas, sociales y culturales de vida de las poblaciones, las cuales determinan y se expresan en los procesos de salud-enfermedad; en la respuesta para su atención incluso definen las formas de morir de los grupos humanos (Blanco et al., 2014; Laurell, 1994; Ortiz \& Staines, 2020).

Se ha señalado que ningún sistema de salud estaba preparado para detener la propagación del contagio. De cara a esta situación, el sector salud en México ha puesto a prueba su capacidad para diseñar y operar estrategias sanitarias que prevengan, controlen y atiendan la infección por el COVID-19. Ante esta respuesta, se han sumado problemas de salud pública como: hipertensión, diabetes mellitus y obesidad que agravan la problemática (Ortiz \& Staines, 2020; Suárez et al., 2020). Y si bien los hombres han enfermado más (52.55\%), comparado con las mujeres (47.45\%), al 28 de agosto de 2020 (Secretaría de Salud [SSA], 2020a), las cargas de enfermedad o daños se expresan diferencialmente en las mujeres.

Frente a este panorama, se suman otros estragos, como la falta de justicia social y laboral, los cuales producen injusticia para la obtención de los satisfactores de vida, entre los cuales destacan el acceso a la educación, la vivienda, la alimentación y la salud, incluyendo la atención médica (Blanco et al., 2014). La reciente pandemia de COVID-19 ha empeorado la vulnerabilidad de distintos grupos poblacionales, esto lleva a reflexionar en qué forma las condiciones de vida determinan la adopción de las medidas sanitarias decretadas en marzo (SSA, 2020b).

En este contexto, la Jornada Nacional de Sana Distancia (SSA, 2020b) recomendó el lavado frecuente de manos; la suspensión de actividades no esenciales y el confinamiento, medidas para contener la velocidad de contagio entre una población tomada como estructuralmente homogénea. A pesar de esto, la heterogeneidad y disparidades sociales entre grupos poblacionales se expresaron. Por ejemplo, el confinamiento de algunas familias ocurrió bajo condiciones de hacinamiento y precariedad (De Sousa Santos, 2020). En cuanto al tipo de empleo, 31 millones de personas con ocupación informal (Instituto Nacional de Estadística y Geografía [INEGI], 2020b) no pudieron prescindir de las actividades diarias que les garantizan un ingreso para su sub- sistencia. En otros casos, la subcontratación, la inestabilidad laboral y la falta de seguridad social provocaron que algunos trabajadores/as se vieran expuestos al contagio para mantener su empleo (Castillo-García, 2020).

Según la Encuesta Telefónica de Ocupación y Empleo (INEGI, 2020a), 9.5 millones de personas suspendieron su trabajo sin goce de sueldo; mientras que 1.2 millones perdieron el empleo, renunciaron o cerraron su fuente de ingreso. Este reporte no señala datos por sexo, pero el Consejo Nacional de Evaluación de la Política de Desarrollo Social ([CONEVAL], 2020) ha destacado las brechas laborales para las mujeres. Comparado con los hombres, ellas obtienen un menor ingreso en situación de pobreza, 73 pesos frente a 100 pesos; y dedican a la semana 27 horas más al trabajo no remunerado. Estas condiciones profundizan su desigualdad social y de género al obtener un menor ingreso económico y/o invertir mayor tiempo en tareas por las que no reciben un salario (Coffey et al., 2020).

Un siguiente punto por reflexionar es cómo la urgencia sanitaria por COVID-19 enfocó su primera respuesta en la infección viral, desde una perspectiva biomédica. Esta acción colocó de forma no paralela la atención de la salud mental y las adicciones; la violencia doméstica, la violencia estructural; los problemas por ansiedad, depresión e ideas e intentos suicidas. Esto a pesar de que de la Organización de las Naciones Unidas ([ONU] 2020a), señaló que, en países de ingreso bajo o medio, entre 76 y $85 \%$ de la población con estos padecimientos no recibe tratamiento.

$\mathrm{Y}$, si bien en el país se han ampliado estrategias para atender esta área de la salud (Alcocer, 2019), es necesario retomar y dar respuesta a los rezagos previos. Las mujeres con problemas por el consumo de alcohol son una muestra de ello, pues tienen menor acceso al tratamiento por dependencia de bebidas alcohólicas, esta diferencia es de una mujer por cada 2.2. hombres (INPRFM, INSP, SS, 2012; Reporte de la sociedad civil sobre consumo nocivo de alcohol, 2020). Los datos son alarmantes ya que el consumo excesivo de alcohol en mujeres se ha incrementado, de $4.1 \%$ a $10.3 \%$ en grupos de edad de 12 a 65 años, y en edades de 12 a 17 años pasó de $2.2 \%$ a $7.7 \%$, al comparar los años 2011 y 2016 (ENCODAT, 2016-2017).

Ante el panorama sanitario actual, la Organización de las Naciones Unidas ([ONU] 2020a); Elbay et al., (2020) Hansel et al. (2020), advierten la exacerbación de daños psicosociales durante o después de la pandemia, entre estos: el incremento de la violencia doméstica, el aumento del estrés, la incertidumbre laboral, el temor a ser contagiado/a sin dejar de lado los procesos de duelo. Estos hechos en la vida de las personas y sus colectivos también aumentarán las conductas no saludables, como 
el consumo excesivo de alcohol y/o tabaco (Comisión Nacional contra las Adicciones [CONADIC], 2020). De acuerdo con Hansel et al. (2020), la evidencia científica demuestra que la salud mental de los grupos sociales se ve impactada en momentos de crisis económicas, sanitarias y ante los desastres naturales.

Estos hechos permiten asumir que la enfermedad mental, si bien tiene una dimensión individual, también tiene una dimensión colectiva, y justo es en estas afectaciones grupales donde se reflejan las desigualdades sociales, la falta de protección social, las brechas en la atención médica e incluso se develan las violencias estructurales, a través de las cuales se imponen e invisibilizan relaciones de abuso, subordinación, opresión, estigmatización y discriminación (Lázaro, 2020; Stolkiner \& Ferreyra, 2020).

En este sentido, se admite que los daños psicosociales, al igual que otros modos de enfermar trascurren bajo los determinantes sociales de la salud, como el género, edad, clase social, pertenencia étnica, ocupación, condición migratoria (Lázaro, 2020; Stolkiner \& Ferreyra, 2020). Concerniente a las mujeres, Burín (2010) aporta que el género, como construcción sociocultural, además de mediar y naturalizar relaciones asimétricas, mandata roles y tareas por cumplir, estas son: funciones e ideales maternales, el trabajo de cuidados y de reproducción social, así como trabajo extradoméstico. Las contradicciones, precariedades, obstáculos y tensiones derivadas de estas normas en la vida de las mujeres pueden experimentarse como problemas de salud mental.

En esta línea argumentativa, y como parte de una investigación que se enfoca en conocer la atención que reciben las mujeres debido a los daños por el consumo de alcohol, se definió el siguiente objetivo: comprender la experiencia de las mujeres que reciben apoyo para su recuperación debido a problemas por el consumo de alcohol en agrupaciones de Alcohólicos Anónimos en el contexto de la pandemia por COVID-19, ya que frente a las medidas sanitarias era de interés saber ¿qué situaciones se encontraban viviendo las mujeres en sus agrupaciones de AA y cómo continuarían su proceso de recuperación?

\section{MÉTODO}

Tipo de investigación. Estudio cualitativo de tipo exploratorio. Pretende la aproximación e interpretación de las acciones y de los sentidos de los grupos sociales, la comprensión de procesos y contextos, tensiones o contradicciones (Martínez-Salgado, 2010).

Participantes. La selección de las participantes sucedió con el muestreo de oportunidad. Martínez-Salgado (2012) dice: "aprovechar lo inesperado", tomar lo emer- gente para aproximarse al problema que se estudia (Creswell, 2007). Los criterios de inclusión fueron mujeres mayores de 20 años que acuden a grupos de AA para su recuperación del alcoholismo.

Se contó con la participación de cuatro mujeres, integrantes de grupos mixtos (hombres y mujeres) y tradicionales (sesionan hora y media) en la Ciudad de México y el Estado de México.

Procedimiento. El primer contacto fue con una mujer que acude a un grupo de Alcohólicos Anónimos, con quien tenía relación la investigadora principal, ella fungió como "portera" (Taylor \& Bodgan, 2000) para acercar a otra mujer, esta última contactó a las demás participantes.

Cabe decir que el confinamiento determinó el uso de la aplicación de mensajería instantánea WhatsApp, por este medio fue enviada la invitación para participar en el estudio, se explicó el objetivo y se adjuntó fotografía del consentimiento informado. Una vez aceptada la colaboración por parte de las participantes y manteniendo el distanciamiento físico, se acordó fecha y hora para la entrevista telefónica. Cada entrevista tuvo una duración de una hora y fueron realizadas del 4 al 28 de mayo de 2020.

Instrumentos. Directorio y entrevista semiestructurada, la cual recabó datos sociodemográficos y recuperó información sobre las experiencias vividas por las mujeres al asistir a las agrupaciones durante el confinamiento. Los temas explorados fueron: acciones de prevención de contagio, situaciones vividas frente a la sana distancia y percepción de sus familiares.

Consideraciones éticas. A cada participante se le leyó el consentimiento informado; se le explicó la importancia de su participación voluntaria y se le pidió autorización para audiograbar la información, asegurando su uso éti$\mathrm{co}$, el anonimato y confidencialidad. Se enfatizó que podían suspender la entrevista en cualquier momento.

Procesamiento de la información. La trascripción-examinación de las entrevistas y notas permitieron identificar interacciones entre integrantes de las agrupaciones, los sentimientos, las percepciones, preocupaciones, acontecimientos y situaciones comunes o diferentes sobre las experiencias. Estas vivencias fueron nombradas/ conceptualizadas, se formaron categorías para contrastarlas o relacionarlas, se compararon anotaciones y se configuraron redes. Se utilizó el software Atlas.ti.

Análisis de la información. Con la información sistematizada se elaboró un análisis temático. Berenguera (2014) lo define como describir y/o interpretar de "qué se habla", comprender quién dice y desde qué situaciones o experiencias se expresa. La interpretación de la información fue abordada con perspectiva de género.

El reconocimiento de los mandatos y roles socioculturales asignados a las mujeres, las situaciones, las expre- 
siones reales/concretas en su vida y relaciones asimétricas dada su condición de género (Scott, 2011) permiten la comprensión de sus vivencias y prácticas dentro de las agrupaciones de AA en un determinado contexto sociohistórico, como el de la pandemia de COVID-19.

\section{RESULTADOS}

Todas las entrevistadas son madres, una tiene hijos/as menores de edad (Tabla 1). Ninguna reportó recaer en el consumo del alcohol. Cada participante pertenece a una agrupación distinta, tres agrupaciones están ubicadas en el Estado de México y una en la Ciudad de México. Para mantener el anonimato se asignó un código a cada una de las mujeres.

Se definieron tres categorías, las cuales buscan recuperar las palabras con las que las mujeres nombran las situaciones vividas. Estas son: 1) Una oportunidad; 2) Cosas del gobierno; 3) Ahí voy a estar hasta que Dios me preste vida.

Tabla 1

Características sociodemográficas de las mujeres entrevistadas

\section{“Una oportunidad”. Mujeres en agrupaciones de Alcohólicos Anónimos-AA.}

Esta categoría contextualiza las situaciones que las mujeres viven para ingresar y permanecer en las agrupaciones. Se inicia por referir la participación diferenciada de las mujeres en los programas de tratamiento del sector público de salud debido a barreras para su atención, como son: escasez de programas basados en sus necesidades en salud, su estigmatización y falta de apoyo familiar para reconocer el abuso del consumo de alcohol como problema de salud. Estas condiciones también se expresan en los programas de ayuda mutua. Dicha situación se confirma en la experiencia de una mujer:

Es que es difícil que la mujer llegue al grupo de Alcohólicos Anónimos, es muy, muy difícil, por ejemplo, de quince, dieciocho compañeros, hay dos...o tres a lo mucho. M1-EDOMEX.

\begin{tabular}{|c|c|c|c|c|}
\hline Código & Edad & $\begin{array}{l}\text { Tiempo en la agrupación } \\
\text { de Alcohólicos Anónimos }\end{array}$ & Escolaridad & Ocupación \\
\hline M1-EDOMEX & 42 & $\begin{array}{l}12 \text { años } \\
\text { Los mismos años } \\
\text { en sobriedad. }\end{array}$ & $\begin{array}{l}\text { Medio superior } \\
\text { incompleto }\end{array}$ & $\begin{array}{l}\text { Empleada, continuó laborando } \\
\text { durante la contingencia } \\
\text { sanitaria por COVID-19. }\end{array}$ \\
\hline M2-EDOMEX & 52 & $\begin{array}{l}27 \text { años } \\
\text { Los mismos años } \\
\text { en sobriedad. }\end{array}$ & Secundaria & $\begin{array}{c}\text { Desempleada a causa de la } \\
\text { contingencia sanitaria } \\
\text { por COVID-19. }\end{array}$ \\
\hline M3-CDMX & 45 & $\begin{array}{l}14 \text { años } \\
\text { Los mismos años } \\
\text { en sobriedad. }\end{array}$ & Primaria & Ama de casa \\
\hline M4-EDOMEX & 51 & $\begin{array}{l}15 \text { años } \\
\text { Los mismos años } \\
\text { en sobriedad. }\end{array}$ & Medio superior & Autoempleo \\
\hline
\end{tabular}


No sólo la disponibilidad de servicios médicos o programas se asocia con el rezago de su atención, también la condición de género influye en esta diferenciación. Mientras la familia o la pareja apoyan a un varón para tratar su consumo de alcohol, a una mujer se le condiciona porque los roles y estereotipos tradicionales les exigen dar prioridad a las tareas relacionadas con el hogar, así como la crianza y el cuidado de los hijos/as. Si las mujeres no cumplen con este mandato, por su consumo abusivo de alcohol o por atender los problemas de salud derivados de éste, entonces son juzgadas. Estas desventajas fueron expresadas por las participantes:

Hay mucha desigualdad, mucha desigualdad, porque si yo tengo un hijo que toma, "vete a tu grupo" y hasta yo misma lo acompaño, para que le den su primera junta de información, no siendo así con la mujer, la mujer es señalada: "quédate con tus hijos", "qué andas ahí de chismosa", "si salen mal, va a ser tu culpa porque no les tomas tiempo, porque estás metida allá en tu grupo". M4-EDOMEX.

Las mujeres refieren que las familias sostienen estas desigualdades, ya que convivir con una mujer que se excede en el consumo de alcohol resulta vergonzoso, incluso es motivo de señalamientos si acuden a su recuperación. El siguiente testimonio evidencia el estigma y la discriminación expresados por la familia:

En las familias es así como que lo peor que una mujer llegue a un grupo de Alcohólicos Anónimos, lo hemos visto, a veces los mismos familiares prefieren tener a las mujeres encerradas, aunque estén tomando, pero encerradas en su casa, antes de que lleguen a un grupo. M3-CDMX.

Cuando las mujeres ingresan a un programa de AA, su proceso de recuperación no se ve como un derecho que les asiste para atender sus problemas de salud, sino que se asume como una oportunidad, siempre y cuando se cumpla con las tareas consideradas femeninas. Los familiares no reconocen que ellas tienen un problema de salud por atender; esta situación las lleva a mantener su proceso de recuperación sin descuidar cargas familiares, que incluyen entregar su tiempo a los demás e invertir el tiempo restante en sus necesidades, reafirmando estereotipos como cumplir con tareas de cuidado y rendir sacrificio.

A mí me dijeron "sacrifícate tú, no a la familia" ¿no? ... lo aprendí. Yo tengo un evento, por ejemplo... ya sé que el domingo porque ya está programado, "yo necesito estar a las diez de la mañana" ok, desde el sábado compro la comida. M2-EDOMEX.
También deben verse como buenas mujeres, mostrarse como dependientes y exclusivas. Esto conlleva a una regulación hacia la mujer, ejercida bajo el poder de la pareja, mal interpretando su interés por cuidarse y temiendo ante la posibilidad de romper con el estereotipo exigido socialmente. En este caso, a las mujeres se les asume como un objeto de control. Así lo expresó una de las entrevistadas:

No tienen la misma oportunidad que el hombre, si tiene novio, que porque el novio "ay, de seguro vas porque vas a buscar un amorcito", si es casada pasa lo mismo, los celos, la inseguridad, o sea no les dan esa confianza y esa oportunidad, no lo ven como algo de salud, no lo ven como un problema, lo ven como ¡Ay, se va a ir a enredar con alguien más! M1-EDOMEX.

Como se identifica en los relatos, las mujeres entrevistadas si bien reciben apoyo para su recuperación en los programas de AA, también pueden llegar a experimentar discriminación por ser mujeres, o bien, la descalificación de sus necesidades de salud. Además, se espera que cumplan con los mandatos tradicionales de género.

\section{"Cosas del gobierno". Las tensiones ante las medi- das de prevención de contagio en los grupos de AA.}

La cotidianidad de las mujeres en las agrupaciones de Alcohólicos Anónimos se vio trastocada por la pandemia de COVID-19. Esta categoría analiza tres tensiones que develan las situaciones diferenciadas entre las y los integrantes de AA frente a la emergencia sanitaria. También se muestra la desacreditación que las mujeres viven al nombrar sus preocupaciones, teniendo que asumir las expresiones con las que sus compañeros imponen sus percepciones.

Como primera tensión se identificó la condición de salud previa; la diabetes, las enfermedades relacionadas con el consumo prolongado de alcohol y la edad de las/los integrantes de AA los colocaron como grupos de riesgo. Posteriormente, se supo de compañeros infectados y algunos otros que fallecieron por COVID-19, lo cual aumentó la dificultad para elaborar los duelos debido a que las restricciones impidieron la asistencia de más de 10 personas a los servicios funerarios. Así lo relataron las mujeres entrevistadas:

Hay grupos que, por ejemplo, tienen gente que están enfermos... tienen bastante gente que tienen diabetes (...) Compañeros que tienen diabetes, pues empezaron a sentirse un poco mal, entonces también el doctor les dijo que tomaran la cuarentena. M1-EDOMEX. 
Por mensaje nos avisaron que ya había fallecido el compañero... entonces bueno, pues ahorita ya no se pudo ni... ir a verlo, ni ir a su velorio, pues.... nada, ya no hubo oportunidad de despedirnos ni nada. M3-CDMX.

Algunas mujeres en AA reconocieron que el contagio podía aumentar entre sus compañeros. El trabajo desempeñado por ellos los exponía a la infección (era mayo y en la Central de Abastos en la CDMX se daba la noticia de un brote por COVID-19). Estos acontecimientos reflejan la condición laboral y falta de seguridad social en salud, desigualdades estructurales manifiestas en la situación de las y los integrantes de AA. Si bien este hecho no afecta directamente a las mujeres entrevistadas, sí les genera angustia que los hombres del grupo estén expuestos.

Sí llegó muy, muy fuerte por acá por estos este... lugares, algunos compañeros porque trabajaban este... en la Central de Abastos, entonces, este... sí creo yo que de repente nos confiamos mucho de que... pues no iba a ser tan grave...

Son jubilados, algunos tienen sus negocios propios, como alguna tiendita, ... ayudantes de algo, pero... son muy, muy pocos los que tendrán algún seguro o algo, porque casi son independientes ¿no?, algunos tienen su taxi de trabajar. M3-CDMX.

La segunda tensión ocurrió cuando las recomendaciones de distanciamiento físico sugirieron suspender las actividades para trasmitir el mensaje en hospitales, iglesias y escuelas, así como para la identificación de "prospectos". Los disensos inician porque para algunos de los integrantes de AA estas recomendaciones contradicen los propósitos de la comunidad de Alcohólicos Anónimos. Mientras algunas agrupaciones cerraban, otras decidieron abrir el lugar de sus reuniones, estos espacios suelen ser locales pequeños que los integrantes rentan para realizar sus sesiones de recuperación y recibir a quien acude por primera vez. Así lo narró una de las entrevistadas:

Ahorita, este... aunque nos llegara información de ir ayudar este... a alguna persona, definitivamente no se puede ¿no?, nosotros por ejemplo trabajamos mucho haciendo visitas, directamente en casas, si el familiar pide la ayuda (...) Nosotros sabemos que... es muy importante que un grupo permanezca abierto, por todas esas necesidades que vamos viviendo en un proceso de recuperación. M3-CDMX.
Las controversias se muestran y se convierten en una tercera tensión entre integrantes de las agrupaciones. La desinformación y confusión caracteriza la disyuntiva, ya que determinan la creencia sobre este nuevo coronavirus y la adopción de las medidas de prevención de contagio. Lo relevante de esta información es la actitud retadora por parte de los hombres para no protegerse, esto incrementó la angustia experimentada por las mujeres y devela la forma diferenciada en que se asumen las recomendaciones. Así lo expresaron:

No se decidió hacer nada porque se creía, o al menos así se llegó a pensar, de algunos compañeros, que eran cosas del gobierno, que eso no nos involucraba a nosotros (...) Veo que hay mucho desconocimiento en las agrupaciones, sobre lo... que es esta pandemia, posiblemente creen más en lo que ven en una publicación del Face o de un mensaje de WhatsApp. (...) decían: "es que hasta que no nos llegue un papel de la autoridad, nosotros no cerramos, a menos que vengan... y eso si nos cierran, pues podemos sesionar, bajamos la cortina". M4-EDOMEX.

También se identificó que la condición de género determinó dos situaciones para las mujeres: la primera es la adopción de las medidas de distanciamiento y la segunda son las diferencias entre hombres y mujeres. Mientras los varones en AA llamaban a las acciones de prevención de contagio como "cosas del gobierno" y expresaban tener inmunidad frente al nuevo coronavirus dada su sobrevivencia al alcoholismo, las mujeres mostraban sensibilidad para seguir las recomendaciones. Así se mostró en los siguientes relatos:

Hay uno que se ríe, me dice: "Ay, por favor, cómo crees en eso, eso nada más es política", le dije "mira, no sé si sea cierto o no, pero más vale" (...) Se creen más fuertes, que no les va a hacer nada eso, que si el alcohol no los mató. M2-EDOMEX.

Bueno yo decidí no saludarlos, que fue algo también que me causó problema dentro del grupo. Incluso no evitan el saludo, porque llega el que se burla y: "¿a poco tienes miedo?". No quisieron ponerse el gel y diciendo: "pues si nos va a cargar la fregada, pues que nos cargue". M4-EDOMEX.

Las mujeres que asisten a AA buscaban seguir las recomendaciones de cuidado y prevención señaladas por las autoridades sanitarias. Asimismo, expusieron preocupación o temor al contagio, lo que definió las acciones que adoptaron, como uso de cubrebocas, gel antibacte- 
rial, etcétera, sin embargo, eso se convirtió en motivo de burla y descalificación. Esto revela estereotipos sobre lo masculino representados por la fuerza, el desafío y la valentía, sobre estereotipos considerados femeninos como la sensibilidad o el temor. Además, a las mujeres se les limitó ejercer un liderazgo para que sus recomendaciones fueran consideradas. Así lo relataron:

Ellos me dijeron, cuando yo les propuse que no sesionáramos, me dijeron, "es que tú no tienes", "nosotros no hemos recibido una orden del gobierno para que nos prohíba cerrar", "tampoco somos treinta, entonces sabes qué, si nosotros decidimos que esto siga que continúe, pues adelante". M2-EDOMEX.

Además, los hombres de estos grupos, visto por las mujeres, tienen dificultad para tomar decisiones que los lleven a cuidar de sí mismos y del resto del grupo.

Que los compañeros, el desafiar, el decir pues "no nos pasa nada", "no hay que tener miedo" (...) Fui yo como mujer, diciendo todo esto que estaba pasando, fui así como el blanco de burlas, tienen miedo. Yo lo veo... que... a pesar de que se dice que estamos en un mundo de iguales, si una mujer hace una propuesta, no es tan... considerada, como... si lo propone un hombre. M4-EDOMEX.

Esta información demuestra las diferenciaciones de las relaciones entre géneros. Las mujeres entrevistadas lidian con la anulación de sus propuestas de cuidado de la salud por la crisis sanitaria, reiterando su subordinación.

\section{"Ahí voy a estar hasta que Dios me preste vida". Ser y sentir en el contexto del COVID-19.}

Esta categoría reveló los sentimientos de las mujeres entrevistadas. La información recolectada dio a conocer que la incertidumbre y el desconcierto sobre el COVID-19 debilitan la toma de decisiones para realizar las medidas de distanciamiento, la desinformación caracterizó el momento. El siguiente testimonio expuso la situación:

Llega la parte de.... pues de sentir miedo, a veces llega la parte de decir ¿qué va a pasar? porque, por un lado, oyes una cosa y por otro lado oyes otra cosa, en la parte de... ¿cuál es lo real? ¿cuál es lo real? y es cuando llega la confusión y lo único que te queda es... nos dice el programa... "agárrate de algo", "algo superior a ti", y eso es lo que nosotros hacemos ¿no?, sea verdad o sea mentira. M1-EDOMEX.
El temor por el contagio del COVID-19, las enfermedades crónicas, la edad como factor de riesgo y la influencia de la familia para que la mujer que cuida de otros no se expusiera al contagio, fueron parte de la determinación de las asistentes a AA para tomar el distanciamiento temporal hacia sus agrupaciones, con lo que disminuyó su participación y se pone en riesgo su recuperación. El reconocimiento de emociones y sentimientos no sólo ayuda a confirmar las diferencias de género para la adopción de las medidas sanitarias, sino también destaca sus desventajas, porque además de lidiar con el cuestionamiento de sus compañeros se vieron enfrentadas al aumento de carga de trabajo en sus hogares. El siguiente relato lo describe:

Yo soy diabética, yo tengo mis... tomo mis medidas, me salgo con cubrebocas, tomo mi sana distancia (...) Las mujeres somos más responsables. M2-EDOMEX.

Les comenté sobre mis miedos, que yo sí tenía miedo ¿no?, y este... y que, pues también mi familia estaba preocupada, yo decidí no seguir asistiendo, "yo me guardo en mi casa". M4-EDOMEX.

Yo tengo a mi madre, mi madre es hipertensa, ella vive en el Estado de México, pero yo me la traje desde que empezó lo de la primera etapa, yo me la traje conmigo este... aquí a la casa donde vivo, entonces, por esa situación fue que yo empiezo a tomar decisiones de no salir, de no irme a ningún lado (...). Yo creo que muchas hemos de estar un poco presionadas, este... sabemos que nosotras como mujeres, cargamos más, con todo eso de los problemas de la familia. M3-CDMX.

La falta de empleo también determinó la situación laboral y económica, tanto de los hombres como de las mujeres que acuden a AA, lo que generó en ellas doble preocupación, la personal y la de los compañeros del grupo. Sin dejar de lado que, para algunas esta pandemia representó volver a depender de sus familiares, por no contar con un salario propio y quedar a expensas del apoyo de los demás.

Aquí en la casa ellos ahorita se van a vender donas, yo no salgo, yo estoy en la casa y.... porque ahorita nadie tiene trabajo, se llevan su cubrebocas, sus guantes, todo bien "en nombre sea de Dios” ¡A darle! M2-EDOMEX.

Una fuente adicional de angustia para las mujeres en AA es el hecho de tener que enfrentar solas el sustento y 
cuidado de ellas y el de sus hijos/as, ya que no cuentan con apoyo de otros familiares frente a la crisis de salud actual. Así lo compartió una mujer:

No tener los recursos para poder este... salir de esta enfermedad, decía "yo no tengo los recursos, yo soy sola, mi hijo todavía depende de mí y yo necesito cuidarme". Muchas se están viendo presionadas, precisamente porque son mamás, la mayoría son mamás solteras. M4-EDOMEX.

El descrédito y el hostigamiento fueron una constante. Descalificar las emociones o percepciones de las mujeres, así como el acoso, determinaron que ellas desistieran de los servicios que realizaban, estas son tareas de responsabilidad y funciones que se asumen para promover la unidad y organización del grupo y la comunidad AA. La información develó que este escenario representa un obstáculo más para su participación en el grupo. Las experiencias lo señalan:

No somos tan escuchadas y tenemos mucho miedo a ser criticadas, por eso no hablamos, no decimos jay, es que yo sí tengo miedo! Yo estaba haciendo un servicio, de hecho, en el cual acabo hasta de renunciar, precisamente porque en las plenarias, que son diferentes al grupo, pues se juntan muchos más compañeros, igual, sin ningún tipo de medida. M4-EDOMEX.

Evidentemente, el contexto de la pandemia de COVID-19 dejar ver que la oportunidad de recuperación es limitada para las mujeres y eso las pone en riesgo, pues surge en ellas la idea de perder la oportunidad de ese espacio para continuar con su recuperación.

La agrupación pasa a ser parte de nuestra vida cotidiana ¿no?, nosotros decimos "una hora y media de reunión, es así como ir por la medicina”. Para mí es indispensable, es indispensable. Me decían mis hijos: "mamá, ahora que termine lo de la pandemia, ¿qué es lo primero que vas a hacer?" y yo me empecé a reír y les dije: "voy a correr a mi grupo" y dijeron: jay, cómo crees!, les dije: "sí, claro que sí..." Entonces, yo creo que esto pasó a ser parte de mi vida, es algo que yo quiero, que me agrada, y pues que ahí voy a estar hasta que Dios me preste vida. M3-CDMX.

Finalmente, la información demostró que asistir a un grupo de AA para la recuperación en un contexto de emergencia sanitaria intensifica las desventajas para las mujeres. La idea de no poder acudir al único lugar que les ha dado la oportunidad para atender sus problemas de salud les genera fuertes tensiones.

\section{DISCUSIÓN Y CONCLUSIONES}

Las mujeres constituyen grupos en vulnerabilidad y la crisis por el COVID-19 acentúa su desigualdad social y de género (De Sousa Santos, 2020; ONU, 2020b).

La comprensión de la salud, la enfermedad y su atención como proceso histórico, social y culturalmente determinado, de cara al COVID-19, permite reconocer la condición de género como un determinante de la situación de las mujeres (Scott, 2011) que viven los daños por el consumo de alcohol y que se encuentran en recuperación, entendiendo, de acuerdo con Rasovsky (2009), que se acude a los programas de ayuda mutua para lograr la sobriedad. Para las mujeres esto no debe ser un hecho menor, primordialmente cuando son ellas quienes enfrentan mayores rezagos para su atención, la condición de género les impone el estigma, la discriminación y la falta de reconocimiento a su consumo de alcohol como un problema de salud, circunstancias señaladas por Romero et al., (2011), Ettorre (1998), Góngora y Leyva (2005). Para las mujeres entrevistadas se está lejos de la posibilidad de optar por un servicio privado.

La información obtenida en este estudio reveló cómo los estereotipos y roles tradicionales de género expresados en las agrupaciones reafirman relaciones asimétricas; esta apreciación ha sido destacada por Cortaza y Villar (2008), Galaviz (2015), pero la crisis sanitaria actual recalca estas desigualdades.

También se señaló que la descalificación y el hostigamiento influyen en el acceso de las mujeres al empleo (Salinas-Urbina, 2012), para el caso expuesto estas expresiones obstaculizaron la participación de las mujeres en sus procesos de recuperación del consumo de alcohol.

Frente al contexto sanitario actual, fue posible destacar que las medidas de prevención de contagio y los cuidados para la salud por los que las mujeres en Alcohólicos Anónimos optaron, fueron menospreciados. Si bien, históricamente se ha socializado a las mujeres para cumplir con funciones como las del cuidado, contradictoriamente sus conocimientos y decisiones no son legitimados.

Se reflexiona que, en tanto se asuma que cuidar es una responsabilidad exclusiva de las mujeres, se asignarán mayores cargas de trabajo no remunerado e invisibilizado, así como preocupaciones. Dichas situaciones condicionan el tiempo para el autocuidado y recuperación, repercutiendo en su salud, este señalamiento es coincidente con Diez et al. (2020).

También es posible reconocer que las mujeres que acuden a AA han tenido menores oportunidades para 
el trabajo remunerado y estable, sin duda esto también acentúa su desigualdad. La afirmación se argumenta en los indicadores reportados por el CONEVAL (2020).

Si bien se suman esfuerzos para prevenir los daños a la salud en el contexto del COVID-19, es posible reflexionar que las personas no necesariamente se apropian de la información, mucho menos se disminuyen las brechas para la atención de mujeres con problemas por consumo de alcohol. Se piensa que es importante seguir visibilizando el fenómeno y retomar la recomendación hecha por la ONU (2020a) para continuar atendiendo a personas con problemas de salud mental e integrar acciones comunitarias con perspectiva de género

\section{FUENTES DE FINANCIAMIENTO}

La presente investigación no fue financiada por ningún organismo ni institución.

\section{CONFLICTOS DE INTERÉS}

Las autoras de este manuscrito declaran que no existen conflictos de interés.

\section{AGRADECIMIENTOS}

A las mujeres en agrupaciones de Alcohólicos Anónimos.

\section{REFERENCIAS}

Alcocer, J. (2019). Necesitamos incrementar la capacitación de recursos humanos para mejorar la salud mental. En Secretaría de Salud. Salud y bienestar. (3 Ed, pp. 6-7).

Berenguera, A., Fernández de Sanmamed. S. M. J., Pons, M., Pujol, E., Rodríguez, D. \& Suara, S. (2014). Escuchar, observar y comprender. Recuperando la narrativa en las Ciencias de la Salud. Aportaciones de la investigación cualitativa. Institut Universitari d'Investigació en Atenció Primaria Jordi Gol.

Blanco, J., López, A., \& Rivera, J. (2014). Calidad de vida, salud y territorio. Desarrollo de una línea de investigación. Universidad Autónoma Metropolitana. Unidad, Xochimilco.

Boichat, G. (1 de abril de 2020). "Hay que aprovechar esta pandemia para hacer un cambio social radical. Entrevista a Joan Benach". En: Dossier Covid-19. Impactos socioculturales de la pandemia. Epidemiologia sociocultural-El Colegio de Sonora. P. 156-169.

Burín, M. (2010). Género y salud mental: construcción de la subjetividad femenina y masculina. En: Burín, M. Género y salud mental. Clase llevada a cabo en la Facultad de Psicología de la Universidad de Buenos Aires, Argentina.

Castillo-García, N. (4 de julio de 2020). Cómo hacer frente al subempleo y desempleo por la pandemia. Comisión universitaria para la Atención de la Emergencia Coronavirus. Ciencia UNAM. https://covid19comisionunam.unamglobal.com/?p=87283\&-
fbclid=IwAR06oQnwWyJmFXvBMMidMKnb3N7-XXQel4fVfIRh4XXTz_zbywylgHWNC8Q.

Coffey, C., Espinoza, P., Harvey, R., Lawson, M., Parvaez, A., Piaget, JK., Sarosi, D., \& Thekkudan, J. (2020). Tiempo para el cuidado. El trabajo de cuidados y la crisis global de desigualdad. OXFAM Internacional. https://oxfamilibrary.openrepository.com/ handle/10546/620928.

Comisión Nacional contra las Adicciones. (2020). Consumo de bebidas alcohólicas durante la Jornada Nacional de Sana Distancia por COVID-19. Secretaría de Salud de México. Disponible en: https://www.gob.mx/cms/uploads/attachment/file/548400/CONADIC_Alcohol_COVID-19.pdf

Consejo Nacional de Evaluación de la Política de Desarrollo Social. (2020). Pobreza y género en México; hacia un sistema de indicadores información 2008-2018 (pp. 1-5). Dirección de Información y Comunicación Social.

Cortaza, L. \& Villar, M. (2008). Surge en mí otra mujer: significados del consumo de alcohol en mujeres mexicanas. Escola Anna Nery, 12(4), 693-98. doi: 10.1590/S1414-81452008000400013

Creswell, J. (2007). Qualitative inquiry and research design. Choosing Among Five Approaches. SAGE Publications.

De Sousa Santos, B. (2020). La cruel pedagogía del virus. CLACSO. Diez, M., Pía, M., Vissichio, F., Amendolaro, R., Carina., J., Muñiz., A. \& Arrúa, L., (2020). Entre la invisibilidad y el estigma: consumo de sustancias psicoactivas en mujeres embarazadas y puérperas de tres hospitales generales de Argentina. Salud Colectiva, 16, e2509. doi: 10.18294/sc.2020.2509.

Elbay, R. Y., Kurtulmuş, A., Arpacıoğlu, S., \& Karadere, E. (2020). Depression, Anxiety, Stress Levels of Physicians and Associated Factors In Covid-19 Pandemics. Psychiatry Research, 290, 113130. doi: 10.1016/j.psychres.2020.113130

Ettorre, E. (1998). Mujeres y alcohol, ¿placer privado o problema público? Narcea.

Galaviz, G. (2015). Mujeres, adicción y rehabilitación. Reflexiones desde la frontera noroeste de México. Salud Colectiva. 11(3), 367-379.

Góngora, P. \& Leyva, M. (2005). El alcoholismo desde la perspectiva de género. El cotidiano. Revista de la realidad mexicana actual, 132, 84-91.

Hansel, T., Saltzman, L., \& Bordnick, P. (2020). Behavioral Health and Response for COVID-19. Disaster Medicine and Public Health Preparedness, 1-7. doi:10.1017/dmp.2020.180

Instituto Nacional de Estadística y Geografía. (2020a). Encuesta Telefónica de Ocupación y Empleo (ETOE). Resultados de mayo 2020. INEGI.

Instituto Nacional de Estadística y Geografía. (2020b). Resultados de la Encuesta nacional de ocupación y empleo cifras durante el primer trimestre de 2020. INEGI.

Instituto Nacional de Psiquiatría Ramón de la Fuente Muñiz, Instituto Nacional de Salud Pública, Secretaría de Salud (2012) Encuesta Nacional de Adicciones 2011: Reporte de Alcohol. INPRFM. http://www.conadic.salud.gob.mx/pdfs/ENA_2011_ ALCOHOL.pdf 
Instituto Nacional de Psiquiatría Ramón de la Fuente Muñiz, Instituto Nacional de Salud Pública, Comisión Nacional contra las Adicciones, Secretaría de Salud. (2017). Encuesta Nacional de Consumo de Drogas, Alcohol y Tabaco 2016-2017: Reporte de Alcohol. INPRFM.

Laurell, C. (1994). Sobre la concepción biológica del proceso salud-enfermedad. En: Rodríguez, M. (coord.). Lo biológico y lo social. Serie de desarrollo de recursos humanos (pp. 1-19). OMS-OPS.

Lázaro, S. (5 de junio de 2020). No es enfermedad: es precariedad. Diagnóstico cultura. https://www.diagnosticocultura.com/ no-es-enfermedad-es-precariedad/?fbclid=IwAROREFZvchWQDApuqOmq-9UFvORSOImEY5FiSFIMXIM67|jJJm63gIXI_Ec.

Martínez-Salgado, C. (2010). Por los caminos de la investigación. Exploraciones narrativas y reflexiones en el ámbito de la salud. Universidad Autónoma Metropolitana-Xochimilco.

Martínez-Salgado, C. (2012). El muestreo en investigación cualitativa. Principios básicos y algunas controversias. Ciencia \& Saúde Colectiva. 17(3), 613-619.

Organización de las Naciones Unidas. (2020a). Policy Brief: COVID-19 and the Need for Action on Mental Health. Executive Summary. Recuperado de: http://coronavirus.onu.org.mx/wp-content/ uploads/2020/05/UN-Policy-Brief-COVID-and-mental-health.pdf. Fecha de acceso: 10 de julio de 2020.

Organización de las Naciones Unidas. (2020b). Poner a las mujeres y a las niñas en el centro de los esfuerzos para recuperarse de COVID-19. https://www.unwomen.org/en/news/stories/2020/4/ statement-sg-put-women-and-girls-at-the-centre-of-efforts-torecover-from-covid19.

Ortiz, H. \& Staines, G. (2020). La epidemia de enfermedades crónicas en México: respuestas desde la medicina social. Historia de dos epidemias anunciadas. En: Salud de nuestros pueblos (pp. 12-15). Asociación Latinoamericana de Medicina Social.

Rasovsky, H. (2009). Alcohólicos Anónimos en México: fragmentación y fortalezas. Desacatos. 29, 13-30.

Reporte de la sociedad civil sobre consumo nocivo de alcohol. (2020). México. Coalición Latinoamericana saludable. NCD Alliance.

Romero, M., Galván, J., Martínez, M. \& Rodríguez, E. (2011). Barreras a tratamiento que enfrentan las mujeres: una aproximación émica y ética. En: M. Romero, M. Medina-Mora, \& C. Rodríguez (Eds). Mujeres y adicciones, (pp. 251-265). CENADIC.

Salinas-Urbina, A. (2012). Diferencias por género: la mirada del personal de la universidad de Guanajuato. En: G. Meza-Lavaniegos. y A. Salinas-Urbina. Mujeres y hombres. Perspectivas sobre relaciones de género en la Universidad de Guanajuato.

Scott, J. (2011). Género: ¿todavía una categoría útil para el análisis? La manzana de la discordia, 6(1), 95-101.

Secretaría de Salud México. (2020a). Información general. Recuperado de: https://coronavirus.gob.mx/datos/

Secretaría de Salud México. (2020b). Jornada Nacional de Sana Distancia. Recuperado de: https://www.gob.mx/cms/uploads/attachment/file/541687/Jornada_Nacional_de_Sana_Distancia.pdf.

Stolkiner, A. \& Ferreyra, J. (3 de julio de 2020). Psicopatologizar la cuarentena. http://lobosuelto.com/psicopatologizar-la-cuarentena-alicia-stolkiner-y-julian-ferreyra/?fbclid=IwAR3PpkSDA-uKhaaeKJ5r|fV4TIWJnHbQpq3J7bAh8h06_2rsaXljEHXy3q8.

Suárez, V., Suarez, M., Oros, S. \& Ronquillo, E. (2020). Epidemiología de COVID-19 en México: del 27 de febrero al 30 de abril de 2020. Revista Clínica Española. doi: 10.1016/j.rce.2020.05.007

Taylor, S. \& Bodgan., R. (2000). Introducción a los métodos cualitativos de investigación. La búsqueda de significados. Paidós. 